\title{
Corticosteroids Treatment for Patients With Coronavirus Disease-2019 (COVID-19) With Different Disease Severity: a Systematic Review and Meta-analysis Protocol
}

\section{Shaofei Lou}

Fifth Affiliated Hospital of Sun Yat-sen University

Kaizhuang Huang ( $13610031612 @ 163 . c o m$ )

Fifth Affiliated Hospital of Sun Yat-sen University https://orcid.org/0000-0002-2740-1387

Jiao Jiang

Fifth Affiliated Hospital of Sun Yat-sen University

Ningjun Li

Fifth Affiliated Hospital of Sun Yat-sen University

\section{Protocol}

Keywords: Coronavirus Disease-2019 (COVID-19), Corticosteroids treatment, systematic review, protocol

Posted Date: January 5th, 2021

DOl: https://doi.org/10.21203/rs.3.rs-138312/v1

License: (c) (i) This work is licensed under a Creative Commons Attribution 4.0 International License.

Read Full License 


\section{Abstract}

Introduction: As the coronavirus disease 2019 (COVID-19) pandemic progresses, identifying effective antiviral agents to treat the COVID-19 is of most urgency. Efficacy and safety of corticosteroids in patients with COVID-19 still are debated. Because high-quality randomized clinical trials (RCTs) on the use of corticosteroids for patients with COVID-19 recently were published recently, we aim to conduct a systematic review and meta-analysis of RCTs on corticosteroids therapy in patients with different disease severity to ascertain the effect on survival.

Methods: We will undertake a comprehensive literature search among PubMed, EMBASE, Cochrane Library, medRxiv, and bioRxiv from their inception onwards to identify relevant RCTs. Two reviewers will independently extract data and conduct risk of bias assessments. The primary outcome is all-cause mortality, mortality of mechanically ventilated patients and patients who did not receive oxygen therapy. Secondary outcomes include need for mechanical ventilation or oxygen therapy and incidence of adverse outcomes. Heterogeneity of the estimates across studies will be assessed. Outcomes will be analyzed to pooled risk ratio and pertinent $95 \%$ confidence interval. A subgroup analysis will be conducted by disease severity to explore the source of heterogeneity. The systematic review and meta-analysis will be presented according to the Preferred Reporting Items for Systematic reviews and Meta-Analyses.

Discussion: This systematic review will provide an overview of the current state of evidence concerning the effect of corticosteroids on survival of patients with COVID-19 depended on the disease severity. Also, this systematic review will show the limitations and strengths of the studies available in the literature, as well as recommendations for future avenues of research will be given.

\section{PROSPERO registration number: CRD42020227740.}

\section{Introduction}

By December 2020, there were more than 80 million people confirmed and over 1.7 million deaths cases of coronavirus disease 2019 (COVID-19) globally. The COVID-19 pandemic probably represents the biggest global crisis faced by public health worldwide so far. The World Health Organization (WHO) named the virus 2019-nCoV (novel coronavirus 2019), which belongs to a different branch of the human severe acute respiratory syndrome CoV (SARS-CoV) and Middle East respiratory syndrome CoV (MERS$\mathrm{CoV}$ ) [1]. At present, there is no effective therapy against this new virus. Identifying effective antiviral agents to treat the COVID-19 is of most urgency.

Previous studies [2-5] suggested that once 2019-nCoV virus enters into humans, it targets the key angiotensin-converting enzyme 2 (ACE2) receptor and replicates in cells, leading to cellular injury or death and releasing pro-inflammatory alarmins. Moreover, the innate immune response can be stimulated, resulting in the activation of alveolar macrophages and complement system. The resultant massive inflammatory response causes progressive deteriorations of alveolar and vascular injuries, microvascular thrombosis, and ventilation-perfusion mismatch. In the late stages of the disease, the systemic 
inflammatory reaction may involve other organs, causing multiple organ dysfunction and death. Corticosteroid treatment, which were used during SARS-CoV and MERS-CoV outbreaks, could have a role in inhibiting lung inflammation, suppressing immune response and pathogen clearance and is considered as one of the potential candidates to treat COVID-19 theoretically.

As of December 25, 2020, 84 studies of corticosteroids for the treatment of COVID-19 have been registered on ClinicalTrials.gov. As the pandemic progresses, the pros and cons of corticosteroid therapy for COVID-19 patients have been a hot issue to be explored. One earlier published prospective metaanalysis of clinical trials developed by the Clinical Characterization and Management Working Group of the World Health Organization (WHO), suggested that among critically ill patients with COVID-19, administration of systemic corticosteroids, compared with usual care or placebo, was associated with lower 28-day all-cause mortality [6]. However, the prospective nature of this meta-analysis implies that it is likely that some important ongoing trails have not been included. In addition, more evidence on the potential role of corticosteroids in a larger group of patients with non-severe COVID-19 is also needed.

Because some high-quality randomized clinical trials (RCTs)[7-9] on the effect of corticosteroids on patients with COVID-19 recently were published, recognizing the urgency of evaluating the efficacy of corticosteroids to guide clinical management, we intend to conduct a systematic review and metaanalysis of RCTs on corticosteroid therapy to evaluate the effect on survival of patients with COVID-19 with different disease severity.

\section{Methods And Analysis}

The study will be delineated according to the recommendations from the Cochrane Collaboration [10] and Preferred Reporting Items for Systematic Review and Meta-Analyses guidelines [11, 12]. The study has been registered on PROSPERO (CRD42020227740) on 23 December 2020. The study is expected to start on 1 January 2021 and be completed on 30 June 2021. Should any amendments to this protocol be necessary, they will be recorded on the PROSPERO platform.

\section{Information sources and search strategy}

A systematic comprehensive search will be performed independently in PubMed, EMBASE, Cochrane Library by two reviewers. In order not to miss potentially important research, pre-print servers including medRxiv, bioRxiv will also be searched. The search strategy will include any RCTs ever performed with corticosteroids in patients with COVID-19 (see the search strategy in supplementary appendix 1). Bibliographies of all included studies and any other relevant reviews or meta-analyses identified via the search will be screened. All databases will be searched from their inception to the search date.

\section{Eligibility criteria}

The following criteria will be used for study inclusion: (1) studies involving patients with acute respiratory failure or pneumonia related to 2019-nCoV infection; (2) random allocation to treatment (corticosteroids 
vs. any comparator with no restrictions on dose or time of administration); (3) published in English. The exclusion criteria include: (1) nonadult patients; (2) lack of data on all outcomes of interest; (3) duplicate publications.

\section{Outcomes}

The primary outcome is all-cause mortality at the longest available follow-up. Co-primary endpoints are mortality of mechanically ventilated patients and patients who did not receive oxygen therapy. The secondary outcomes include: (1) need for mechanical ventilation; (2) need for oxygen therapy; (3) incidence of adverse outcomes. Details of the definitions and measurement of serious adverse events will be will be determined after preliminary literature review and data collection.

\section{Study selection and data extraction}

All records identified in the databased will be collected in the reference management software EndNote X8 (Thomson Reuters, New York, NY), and deduplication will be performed. Upon deduplication, records will be screened in two stages. Firstly, the title and the abstract of all records will be screened against the aforementioned eligibility criteria. Secondly, full texts of all articles not excluded in the first stage will be reviewed to determine whether all relevant criteria are met. Both stages will be performed independently by two reviewers. Disagreement will be resolved by consensus. If no consensus can be reached, disagreement will be resolved by adjudication of a designated third reviewer. We will extract the following variables: authors, publication year, study design, population, treatment details (including corticosteroid use, types and doses of corticosteroids, and other treatments), and outcome measures such as mortality, need for mechanical ventilation or oxygen therapy, length of stay and adverse reactions to corticosteroids. We will present the process of search and study selection using a flow process chart.

\section{Quality assessment}

The internal validity and risk of bias of included studies will be evaluated by two reviewers independently, and the level of agreement between them will be recorded. Any disagreements between the two reviewers will be resolved by discussion with a third reviewer. The risk of bias assessment tool developed by the Cochrane collaboration [13] will be utilized to assess the quality of included studies. Risk of bias and quality scores will be presented in a table.

\section{Statistical analysis}

The meta-analysis of comparable data will be performed using Stata version 15.0 software. Binary outcomes from individual studies will be analyzed to compute individual and pooled risk ratio (RR) and pertinent $95 \%$ confidence interval $(\mathrm{Cl})$ by means of inverse variance method. Statistical heterogeneity across studies will be assessed by the Cochrane $Q$ test and quantified by calculating $\mathrm{I}^{2}$. An $\mathrm{I}^{2}$ of $>50 \%$ will be considered to indicate substantial heterogeneity. The random-effects model or the fixed-effect model will be used depending on the heterogeneity of studies included. The pooled RR with $95 \% \mathrm{Cl}$ will be given for either the fixed-effect model or the random-effects model. Sensitivity analyses will be performed by sequentially removing each study and reanalyzing the remaining dataset by analyzing only data from 
studies with low risk of bias and by analyzing studies with low heterogeneity. In addition, we will conduct a subgroup analysis by disease severity to explore the source of heterogeneity. Severity of COVID-19 is based on American Thoracic Society guidelines [14] and the National Guidelines of China [15]. The potential for publication bias will be assessed by funnel plots.

\section{Discussion}

This systematic review will provide an overview of the current state of evidence concerning the effect of corticosteroids on survival of patients with COVID-19 depended on the disease severity. As the COVID-19 pandemic progresses, the primary outcomes analyzed will provide evidence for helping guide clinicians and researchers to understand the safety and efficacy of corticosteroids accurately. Secondary, outcomes will provide information to help government, medical institutions and clinicians to adjust and update the therapeutic schedules of COVID-19 in a timely manner. A decreased timespan from evidence to clinical practice is particularly important in this international healthcare crisis. Furthermore, this systematic review will show the limitations and strengths of the studies available in the current literature, as well as it will to encourage the financing of new studies by public health managers and governmental entities. Strengths of this systematic review include the search in multiple databases, the systematic approach including screening, data extraction, and quality assessment by two independent reviewers, as well as transparency in reporting according to guidelines. The main limitation is the language restriction to English. Only English articles will be included in our study, resulting to the fact that relevant data from non-English spoken areas may lose, and this may cause publication bias to some extent.

\section{Abbreviations}

COVID-19: coronavirus disease 2019; WHO: World Health Organization; 2019-nCoV: novel coronavirus 2019; SARS-CoV: severe acute respiratory syndrome coronavirus; MERS-CoV: Middle East respiratory syndrome coronavirus; ACE2: angiotensin-converting enzyme 2; RCTs: randomized clinical trials; RR: risk ratio; $\mathrm{Cl}$ : confidence interval

\section{Declarations}

\section{Authors' contributions}

$\mathrm{KH}, \mathrm{SL}, \mathrm{JJ}$ and NL drafted the protocol. All authors commented on the manuscript throughout its development. All authors approved the final version.

\section{Funding}

Not applicable.

\section{Availability of data and materials}


Not applicable.

\section{Ethics approval and consent to participate}

Not applicable.

\section{Consent for publication}

Not applicable.

\section{Competing interests}

The authors declare that they have no competing interests.

\section{References}

1. Zhu N, Zhang D, Wang W, et al. A Novel Coronavirus from Patients with Pneumonia in China, 2019. N Engl J Med. 2020. 382(8): 727-733.

2. Huang C, Wang Y, Li X, et al. Clinical features of patients infected with 2019 novel coronavirus in Wuhan, China. Lancet. 2020. 395(10223): 497-506.

3. Ciceri F, Beretta L, Scandroglio AM, et al. Microvascular COVID-19 lung vessels obstructive thromboinflammatory syndrome (MicroCLOTS): an atypical acute respiratory distress syndrome working hypothesis. Crit Care Resusc. 2020. 22(2): 95-97.

4. Feng W, Zong W, Wang F, Ju S. Severe acute respiratory syndrome coronavirus 2 (SARS-CoV-2): a review. Mol Cancer. 2020. 19(1): 100.

5. Bourgonje AR, 0000-0001-5754-3821 AO, Abdulle AE, et al. Angiotensin-converting enzyme 2 (ACE2), SARS-CoV-2 and the pathophysiology of coronavirus disease 2019 (COVID-19). J Pathol. 2020. 251(3): 228-248.

6. JAC S, Murthy S, Diaz JV, et al. Association Between Administration of Systemic Corticosteroids and Mortality Among Critically III Patients With COVID-19: A Meta-analysis. JAMA. 2020. 324(13): 13301341.

7. Liu J, Zhang S, Dong X, et al. Corticosteroid treatment in severe COVID-19 patients with acute respiratory distress syndrome. J Clin Invest. 2020. 130(12): 6417-6428.

8. Angus DC, Derde L, Al-Beidh F, et al. Effect of Hydrocortisone on Mortality and Organ Support in Patients With Severe COVID-19: The REMAP-CAP COVID-19 Corticosteroid Domain Randomized Clinical Trial. JAMA. 2020. 324(13): 1317-1329.

9. Dequin PF, Heming N, Meziani F, et al. Effect of Hydrocortisone on 21-Day Mortality or Respiratory Support Among Critically III Patients With COVID-19: A Randomized Clinical Trial. JAMA. 2020. 324(13): 1298-1306.

10. Cumpston $\mathrm{M}, \mathrm{Li}$ T, Page MJ, et al. Updated guidance for trusted systematic reviews: a new edition of the Cochrane Handbook for Systematic Reviews of Interventions. Cochrane Database Syst Rev. 
2019. 10: ED000142.

11. Moher $D$, Shamseer $L$, Clarke $M$, et al. Preferred reporting items for systematic review and metaanalysis protocols (PRISMA-P) 2015 statement. Syst Rev. 2015. 4(1): 1.

12. Liberati A, Altman DG, Tetzlaff $\mathrm{J}$, et al. The PRISMA statement for reporting systematic reviews and meta-analyses of studies that evaluate healthcare interventions: explanation and elaboration. BMJ. 2009. 339: b2700.

13. JAC S, 0000-0001-8496-6053 AO, Savović J, et al. RoB 2: a revised tool for assessing risk of bias in randomised trials. BMJ. 2019. 366: 14898.

14. Metlay JP, 0000-0003-2259-6282 AO, Waterer GW, et al. Diagnosis and Treatment of Adults with Community-acquired Pneumonia. An Official Clinical Practice Guideline of the American Thoracic Society and Infectious Diseases Society of America. Am J Respir Crit Care Med. 2019. 200(7): e45e67.

15. Lin L, Li TS. [Interpretation of "Guidelines for the Diagnosis and Treatment of Novel Coronavirus (2019-nCoV) Infection by the National Health Commission (Trial Version 5)"]. Zhonghua Yi Xue Za Zhi. 2020. 100(11): 805-807.

\section{Supplementary Files}

This is a list of supplementary files associated with this preprint. Click to download.

- Appendix1.pdf

- PRISMAPchecklist.pdf 